\title{
Inhomogeneity reconstructions in tendon ducts via boundary integral equations
}

\author{
Fatih Yaman ${ }^{\text {a,* }}$, Thomas Weiland ${ }^{\mathrm{b}}$ \\ a Electrical and Electronics Engineering Department, Izmir Institute of Technology, 35430 Urla, Izmir, Turkey \\ ${ }^{\mathrm{b}}$ Institut für Theorie Elektromagnetischer Felder, Technische Universität Darmstadt, 64289 Darmstadt, Germany
}

\section{A R T I C L E I N F O}

\section{Article history:}

Received 24 May 2014

Received in revised form

16 August 2014

Accepted 21 August 2014

Available online 3 September 2014

Keywords:

Inverse scattering problems

Boundary integral equations

Non destructive testing

Buried object detection

Acoustic tomography

\begin{abstract}
A B S T R A C T
In this study, as an alternative to the formerly presented investigations, Newton-type numerical algorithms are proposed to find location and shape of an air void inside of a tendon duct and to identify gathered metallic bars in a concrete column. The simulated structures are illuminated by four acoustic sources at a fixed frequency such that the scattered field is measured in a near-field region at 128 points. According to the nature of physical problems, the Dirichlet boundary condition is employed to model air-filled cavities and transmission conditions are assumed for metallic objects. Additionally, conductive boundary conditions are suggested for a more realistic representation of the inhomogeneities for the rusty metallic skin of the duct. Potential approaches are used to derive boundary integral equations. The proper treatment of the ill-conditioned equations is established via Tikhonov regularization. Applicability of the proposed inversion algorithms is tested with realistic parameters for different scenarios using noisy scattered field data and accurate numerical results are presented at $10 \mathrm{kHz}$ for the unknown physical properties of the duct's skin.
\end{abstract}

(c) 2014 Elsevier Ltd. All rights reserved.

\section{Introduction}

Post-tensioned tendon ducts are commonly used in the reinforcement of the buildings, bridges, stadiums, solid anchors, etc., although they may contain inhomogeneities due to grouting defects, deterioration, overload and aging. These complications can affect the stability of the complete structure. Therefore, nondestructive inspection of ducts plays a critical role in the quality assurance of constructions.

In this study, we investigate applications such that scattered field around the duct can be collected on a closed measurement line e.g. tests of ducts before their installations to the constructions or bridges which include external post-tensioned cables, see Bore et al. [1]. In this context, we consider to identify an air-filled cavity in a post-tensioned tendon and a structural deformation of metallic bars inside of a concrete duct with Newton-type iterative methods. Such methods provide accurate reconstructions of obstacles at a fixed frequency in a resonance region i.e. the operating wavelength and the diameters of the searched objects are of comparable size. Some initial results of reduced model problems, e.g. for a single buried scatterer and a given conductivity

\footnotetext{
* Correspondence to: Izmir Yuksek Teknoloji Enstitusu, 35430 Urla, Izmir, Turkey. Tel.: +902327506596.

E-mail address: fatihyaman@iyte.edu.tr (F. Yaman).
}

function, were recently presented by the authors in [2]. Within this framework, we extend and improve the applicability of our algorithms in the current paper for more complicated scenarios with the realistic parameters for the unknown conductivity function in the reconstructions.

Numerical modeling of elastic waves and validations of theoretical results with the experimental ones are widely studied in the literature due to the importance of non-destructive testing of concrete structures. Among these, acoustic tomography techniques based on the calculation of wave traveling time are frequently employed in practice [3-12]. For the numerical investigation of elastic waves Elastodynamic Finite Integration Technique (EFIT) can be used $[13,14]$ which depends on the adaptation of Weiland's [15] Finite Integration Technique. EFIT is employed for reinforced concrete structures which contain a metallic duct filled with mortar and Elastodynamic Fourier Transform Synthetic Aperture Focusing Technique (EL-FT-SAFT) is applied to image a concrete specimen [16]. SAFT consists of the collection of echo signals over a specific aperture to obtain a reconstruction by performing time shifting and superposition of adjacent signals $[17,18]$. In this direction, Schickert et al. applied SAFT for the reconstruction of ducts in concrete [19]. Furthermore, Langenberg et al. proposed a second inversion method using Born approximation and acoustic waves in 2006 [20].

Recently, Bozza et al. [21] developed no-sampling linear sampling method (nLSM) for electromagnetic fields in order to reconstruct cracks inside a slab, and Agarwal et al. [22] applied 
Multiple Signal Classification to detect reinforcement bars and empty ducts in circular columns.

In this study, a boundary integral equation method based on potential approach is proposed not only for the identification of a void in a duct which contains separate steel bars but also for the problem whose aim is to determine geometrical properties of an arbitrarily shaped metallic structure in a concrete cylinder having a corrosion on its metallic skin. The fields which appear after the interaction of the time-harmonic acoustic plane wave with the scatterers are expressed by boundary integrals containing density (source) functions. To find the density functions we derive a system of boundary integral equations via boundary conditions and jump relations. Density functions and related integral representations allow us to calculate the scattered and the total fields in each region. Then we employ iterative reconstruction algorithms introduced in $[24,23]$ which were tested for shape reconstructions of objects in free-space [24-26] and for the ones buried in bounded domains $[27,28]$.

\section{Problem statement}

Let us consider $D_{0} \subset \mathbb{R}^{2}$ be a bounded medium closured by a smooth curve $\Gamma_{0}$. $D_{1}$ denotes a bounded medium with a smooth boundary $\partial D_{1}$ connected to an interior boundary $\Gamma_{0}$ and arbitrary number of curves $\Gamma_{C_{1}}, \Gamma_{C_{2}}, \ldots, \Gamma_{C_{n}}$, which are indicated by dark colored domains $D_{C_{n}}$ in Fig. 1 , such that $\partial D_{1}=\Gamma_{0} \cup \Gamma_{1} \cup \Gamma_{C_{1}} \cdots \cup$ $\Gamma_{C_{n}}$ and $\Gamma_{0} \cap \Gamma_{1} \cap \Gamma_{C_{1}} \cdots \cap \Gamma_{C_{n}}=\varnothing$. The unbounded domain $D_{2}$ is connected through $\Gamma_{1}$ to the domain $D_{1}$. We shall denote the unit normal by $\nu_{1}$ to the boundary $\Gamma_{1}$ which is directed into exterior of $D_{1}$, and unit normals by $\nu_{0}$ to the boundary $\Gamma_{0}$ and by $\nu_{C_{n}}$ to the boundary $\Gamma_{C_{n}}$ which are directed into exterior of $D_{0}$ and $D_{C_{n}}$, respectively.

In the depicted geometry, we assume cylinders are illuminated by a time harmonic acoustic plane wave $u^{i}=e^{i\left(k_{2} x \cdot d-\omega t\right)}$, where $d=\left(\cos \phi_{0}, \sin \phi_{0}\right)$ is the propagation direction with the angle $\phi_{0}, k_{2}$ is the wave number of the exterior domain, and $\omega=2 \pi f_{0}$ is the frequency and $t$ is the time. However, time factor $e^{-i \omega t}$ is omitted throughout the paper for the sake of the simplicity of the formulations.

In this configuration, total fields $u_{j}$ satisfies the homogeneous Helmholtz equations

$\Delta u_{j}+k_{j}^{2} u_{j}=0$ in $D_{j}$,

with the wave number $k_{j}$, in the corresponding domain $D_{j}$ where $j=\{0,1,2\} \vee\left\{C_{1}, C_{2}, \ldots, C_{n}\right\}$.

We pose conductive boundary conditions $[29,30]$

$u_{2}=u_{1} \quad$ and $\quad \frac{\partial u_{2}}{\partial \nu_{1}}-\frac{\partial u_{1}}{\partial \nu_{1}}=\lambda u_{2}$ on $\Gamma_{1}$,

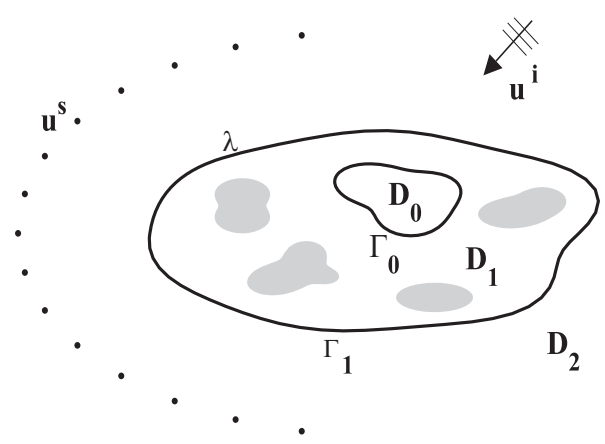

Fig. 1. Geometry of the model problem. with $\lambda$ being a complex valued conductivity smooth function and transmission conditions

$u_{1}=u_{j} \quad$ and $\quad \frac{\partial u_{1}}{\partial \nu_{j}}=\frac{\partial u_{j}}{\partial \nu_{j}}$ on $\Gamma_{j}$,

where $j=\left\{C_{1}, C_{2}, \ldots, C_{n}\right\}$. Depending on the problem type, the Dirichlet or transmission boundary conditions

$u_{1}=0 \quad$ or $\left\{\begin{array}{l}u_{1}=u_{0} \\ \frac{\partial u_{1}}{\partial \nu_{0}}=\frac{\partial u_{0}}{\partial \nu_{0}}\end{array} \quad\right.$ on $\Gamma_{0}$,

are defined on the boundary of the buried object which is to be reconstructed.

The scattered field $u^{s}=u_{2}-u^{i}$ appears due to interaction of the incident wave with the objects and satisfies the Sommerfeld radiation condition at infinity

$\lim _{r \rightarrow \infty} \sqrt{r}\left(\frac{\partial u^{s}}{\partial r}-i k_{2} u^{s}\right)=0, \quad r=|x|$.

In the numerical solution we employ layer potentials to represent the fields and then to derive system of integral equations via boundary conditions and jump relations [32-34]. In order to define layer potentials, let $\Gamma_{j}$ and $\Gamma_{\ell}$ be closed curves and $f$ be a given integrable function. Then the single-layer operator $S_{j \ell, m}: C\left(\Gamma_{j}\right) \rightarrow$ $C\left(\Gamma_{\ell}\right)$

$\left(S_{j \ell, m} f\right)(x):=2 \int_{\Gamma_{j}} \Phi_{m}(x, y) f(y) d s(y), \quad x \in \Gamma_{\ell}$

and its normal derivative $K_{j \ell, m}^{\prime}:=\left(\partial / \partial \nu_{\ell}\right) S_{j \ell, m}$ are defined for $j, \ell$, $m=\left\{0,1,2, C_{n}\right\}$. Here, $\Phi_{m}(x, y)$ is the fundamental solution to the two-dimensional Helmholtz equation, in the domain $D_{m}$ in terms of Hankel function $H_{0}^{(1)}$ of the first kind and zero order

$\Phi_{m}(x, y):=\frac{i}{4} H_{0}^{(1)}\left(k_{m}|x-y|\right), \quad x \neq y$.

We assume boundary curves are parametrized as $\Gamma_{j}=\left\{r_{j}(t) e(t): t \in\right.$ $[0,2 \pi)\}, j=\{0,1\}$, where $e(t)=(\cos t, \sin t)$ is the unit circle.

Even though the scope of the paper does not cover detailed investigations of direct scattering problems with certain numerical methods a few items about our forward solver are remarked in the following. The aim of direct problem here is to find scattered field such that the total fields satisfy the homogeneous Helmholtz equations (2.1) in the corresponding background medium and the related boundary conditions (2.2)-(2.4) under the Sommerfeld radiation condition (2.5). In our numerical solution, fields are represented by combined single- and double-layer potentials and these integral representations are substituted into the boundary conditions to obtain a system of equations whose unknowns are the density (source) functions defined on the curves. Afterwards, scattered field data is calculated on a measurement circle through density functions. As an advantage of the method, an exponential convergence is obtained as it was exhibited in the several publications, see $[32,27,30]$. We refer to the paper [27] for a more detailed presentation of forward solver and for the ideas on the test cases for the accuracy.

In this paper, we study two different geometries which lead us to two types of inverse problems.

IP 1: It is assumed that the domain $D_{1}$ with a conductive boundary $\Gamma_{1}$ contains a sound-soft object $\Gamma_{0}$, and $N$ additional different penetrable objects with smooth boundaries, $\Gamma_{C_{n}}$ $n=1,2 \ldots, N$. The aim of the inverse problem is to find location and shape of the small sound-soft object from the knowledge of the scattered near field for known shapes $\Gamma_{1}, \Gamma_{C_{n}}$ and wave numbers $k_{1}, k_{2}, k_{C_{n}}$.

IP 2: We consider a single penetrable object having transmission conditions on its boundary $\Gamma_{0}$ is buried in $D_{1}$ with a 
conductive boundary $\Gamma_{1}$. The inverse problem here is to find the shape of $\Gamma_{0}$ from the knowledge of the scattered field for known shape $\Gamma_{1}$ and wave numbers $k_{0}, k_{1}, k_{2}$.

\section{Inversion methods}

\subsection{Location reconstruction algorithm for IP1}

Locations of the sound-soft buried objects are found by a simple testing algorithm which depends on the finding minimum value of the residue $\xi$

$\xi=\frac{\left\|u^{s}-u_{(a, b)}^{s}\right\|}{\left\|u^{s}\right\|}, \quad(a, b) \in D_{1}$,

where $u^{s}$ is the scattered field for the problem geometry under investigation and $u_{(a, b)}^{s}$ are the scattered fields obtained by placing an initially guessed cylinder at different locations inside of $\Gamma_{1}$. In a more elaborate way, the domain $D_{1}$ is discretized uniformly by a two-dimensional cartesian grid and direct scattering problems are solved for an initial guess whose center coincides with grid point $(a, b)$.

On the other hand, one can use a Newton iterative type algorithm presented in [27] as an alternative approach for the location reconstructions if a priori information exists on the approximate location and size of the unknown buried object.

\subsection{Shape reconstruction algorithms}

For the inverse problems, we represent the fields in terms of single layer potentials, see [32-35]

$$
\begin{aligned}
u^{s}(x)= & \int_{\Gamma_{1}} \Phi_{2}(x, y) \varphi_{1}(y) d s(y) \text { in } D_{2}, \\
u_{1}(x)= & \int_{\Gamma_{1}} \Phi_{1}(x, y) \psi_{1}(y) d s(y)+\int_{\Gamma_{0}} \Phi_{1}(x, y) \varphi_{0}(y) d s(y) \\
& +\sum_{n=1}^{N} \int_{\Gamma_{C_{n}}} \Phi_{C_{n}}(x, y) \varphi_{C_{n}}(y) d s(y) \text { in } D_{1},
\end{aligned}
$$

$u_{0}(x)=\int_{\Gamma_{0}} \Phi_{0}(x, y) \psi_{0}(y) d s(y)$ in $D_{0}$,

$u_{C_{n}}(x)=\int_{\Gamma_{C_{n}}} \Phi_{C_{n}}(x, y) \psi_{C_{n}}(y) d s(y)$ in $D_{C_{n}}$

Note that as an immediate consequence of the IP1 configuration states that the field $u_{0}$ expressed in (3.4) vanishes due to the Dirichlet condition on $\Gamma_{0}$, and for IP2 the integral representation of $u_{1}$ in (3.3) reduces to the following expression:

$$
\begin{aligned}
u_{1}(x)= & \int_{\Gamma_{1}} \Phi_{1}(x, y) \psi_{1}(y) d s(y) \\
& +\int_{\Gamma_{0}} \Phi_{1}(x, y) \varphi_{0}(y) d s(y) \text { in } D_{0},
\end{aligned}
$$

such that the fields $u_{C_{n}}$ given in (3.5) vanish.

We apply two step algorithms in the solution of IP1 and IP2. The aim of the first step in both algorithms is the reconstruction of the density $\varphi_{1}$ from the solution of an ill-posed data equation via Tikhonov regularization such that

$\alpha \varphi_{1}+S_{11,2}^{*} S_{11,2} \varphi_{1}=S_{11,2}^{*} u^{s}$,

$\alpha$ is a positive parameter and $S_{11,2}^{*}$ is the adjoint operator of $S_{11,2}$.

(i) Shape reconstruction for IP 1: The second step for the solution of IP1 is finding the rest of unknown densities $\psi_{1}, \varphi_{0}, \varphi_{C_{n}}$ from regularized solution of the following system of equations:

$$
\begin{aligned}
& S_{11,1} \psi_{1}+S_{01,1} \varphi_{0}+\sum_{n=1}^{N} S_{C_{n} 1,1} \varphi_{C_{n}}=\left.2 u^{i}\right|_{\Gamma_{1}}+S_{11,2} \varphi_{1} \\
& K_{11,1}^{\prime+} \psi_{1}+K_{01,1}^{\prime} \varphi_{0}+\sum_{n=1}^{N} K_{C_{n} 1,1}^{\prime} \varphi_{C_{n}} \\
& =\left.2 \frac{\partial u^{i}}{\partial \nu_{1}}\right|_{\Gamma_{1}}-\left.2 \lambda u^{i}\right|_{\Gamma_{1}}+\left(K_{11,2}^{\prime-}-\lambda S_{11,2}\right) \varphi_{1}
\end{aligned}
$$$$
S_{1 C_{n}, 1} \psi_{1}+S_{0 C_{n}, 1} \varphi_{0}+S_{C_{n} C_{n}, 1} \varphi_{C_{n}}-S_{C_{n} C_{n}, C_{n}} \psi_{C_{n}}
$$$$
+\sum_{\substack{p=1 \\ p \neq n}}^{N}\left(S_{C_{p} C_{n}, 1} \varphi_{C_{p}}-S_{C_{p} C_{n}, C_{n}} \psi_{C_{p}}\right)=0
$$$$
K_{1 C_{n}, 1}^{\prime} \psi_{1}+K_{0 C_{n}, 1}^{\prime} \varphi_{0}+K_{C_{n} C_{n}, 1}^{-} \varphi_{C_{n}}-K_{C_{n} C_{n}, C_{n}}^{+} \psi_{C_{n}}
$$$$
+\sum_{\substack{p=1 \\ p \neq n}}^{N}\left(K_{C_{p} C_{n}, 1}^{\prime} \varphi_{C_{p}}-K_{C_{p} C_{n}, C_{n}}^{\prime} \psi_{C_{p}}\right)=0
$$

which can be obtained by using conductive boundary conditions (2.2) on $\Gamma_{1}$ and transmission conditions (2.3) on $\Gamma_{C_{n}}$. Here, for the brevity of the notation, we defined the operators

$K_{j \ell, m}^{\prime \pm}=K_{j \ell, m}^{\prime} \pm I$.

Once the density functions are obtained, then the total field $u_{1}$, and its derivative on an initially guessed boundary $\Gamma_{0}$, can be calculated. Afterwards, a sufficiently small $2 \pi$-periodic function $q:[0,2 \pi) \rightarrow \mathbb{R}$

$u_{1}+\left(\operatorname{grad} u_{1} \cdot e\right) q=0 \quad$ on $\Gamma_{0}$,

can be found by evaluating a first-order Taylor expansion in the sense of least squares. Then the update step is performed via $\Gamma_{0}^{u p d}=\left\{\left(r_{0}+q\right) e\right\}$, see [24,27].

(ii) Shape reconstruction for IP 2: In the second step of IP2, we seek for updates not only for the initially guessed shape but also for the density functions. To do this, we consider the following nonlinear operator equation:

$A_{\Gamma_{0}} \chi=b$

where

$A_{\Gamma_{0}}=\left(\begin{array}{ccc}S_{11,1} & 0 & S_{01,1} \\ K_{11,1}^{\prime+} & 0 & K_{01,1}^{\prime} \\ S_{10,1} & -S_{00,0} & S_{00,1} \\ K_{10,1}^{\prime} & -K_{00,2}^{\prime+} & K_{00,0}^{\prime}\end{array}\right)$,

and

$\chi=\left(\psi_{1}, \psi_{0}, \varphi_{0}\right)^{\top}$,

$b=\left(2 u_{\Gamma_{1}}^{i},\left.2 \frac{\partial u^{i}}{\partial \nu_{1}}\right|_{\Gamma_{1}}-\left.2 \lambda u^{i}\right|_{\Gamma_{1}}, 0,0\right)^{\top}$

After the linearization we solve the equation

$A_{\Gamma_{0}} \zeta+A_{\Gamma_{0}, \chi}^{\prime} q=b-A_{\Gamma_{0}} \chi$

and update the densities as well as the shape, i.e. $\chi=\chi+\zeta$, $r_{0}=r_{0}+q$. Here, $A_{\Gamma_{0}, \chi}^{\prime}$ denotes the Fréchet derivative of the operator $A_{\Gamma_{0}}[23,31]$.

The ill-posed behavior of Eq. (3.15) is treated by Tikhonov regularization. For the proper numerical calculations of the singular parts of the operators we refer to [32]. 


\section{Applications and results}

In this section we describe the connection between investigated model problems and practical applications, and present the numerical results for the proposed reconstruction algorithms.

To model non-destructive testing scenarios of tendon ducts, we consider the domains $D_{1}$ and $D_{2}$, which are depicted in Fig. 1, as concrete-type structures such that acoustic waves travel with a speed of $c_{1}=3500 \mathrm{~m} / \mathrm{s}$ and $c_{2}=3000 \mathrm{~m} / \mathrm{s}$, respectively. The wave speed in metal considered as $c_{m}=5000 \mathrm{~m} / \mathrm{s}$. Physically speaking, air behaves highly reflective to acoustic waves and metallic regions permit penetration of acoustic fields. Therefore, using the Dirichlet boundary condition for an air-filled object and transmission conditions for the metallic wires and concrete seems reasonable. Furthermore, a conductivity function is considered on the boundary of the exterior cylinder $\Gamma_{1}$ to model the irregular penetration of acoustic fields to the interior domain of the duct.

In the numerical simulations, exterior cylinder $\Gamma_{1}$ is chosen as a circular cylinder with a radius $r_{0}=19 \mathrm{~cm}$, whereas buried object $\Gamma_{0}$ is considered to be one of the following arbitrarily shaped starlike parametrized curves in the form of $r^{\{a, b, c\}}(t)(\cos t, \sin t)$ where

$r^{a}(t)=7.5 \times 10^{-3}(3.5-\sin t+0.5 \sin 2 t+\cos 3 t)$,

$r^{b}(t)=b_{0} \frac{0.5+0.4 \cos t+0.1 \sin 2 t}{1+0.7 \cos t}, \quad b_{0}=\{0.05,1\}$,

$r^{c}(t)=0.02(6-\sin 2 t+\cos 3 t)$ and $t \in(0,2 \pi]$.

It is assumed that objects are insonificated by an acoustic plane wave at a fixed frequency $f_{0}=10 \mathrm{kHz}$ in four different angles $\phi_{0}=\{0,90,180,270\}$. The noisy scattered near field is calculated on a measurement circle $\Omega_{m}$ with a radius of $r_{m}=25 \mathrm{~cm}$ at 128 equidistant collocation points in each illumination case separately. The contributions of all different scattered fields are taken into account in the construction of a single matrix equation system step of the inversion algorithms. The system of equations is solved via the Nyström method, see [32].

In order to obtain a synthetic noisy field $\tilde{u}^{s}$, random errors are added pointwise to the exact field data $u^{s}$ as follows:

$\tilde{u}^{s}=u^{s}+\eta \delta \frac{\left\|u_{s}\right\|}{\|\delta\|}$

where $\delta \in \mathbb{C},\{\operatorname{Re} \delta, \operatorname{Im} \delta\} \in(0,1)$ denotes a random variable. To have a noise level of $3 \%$ or SNR $\approx 34 \mathrm{~dB}$ the parameter $\eta$ is chosen as $\eta=0.03$. All reconstructions are obtained with noisy data under the assumption of lack of information on the rusty skin of the cylindrical duct. This means that $\lambda=0$ on $\Gamma_{1}$ is considered for the solution of inverse problems although the scattered fields are calculated for conductivity functions $\lambda=\lambda_{1}$ or $\lambda=\lambda_{2}$ such that $\lambda_{1}(t)=0.5$ $(\sin t+i \cos t), \lambda_{2}(t)=\sin ^{4}(t / 2)+i \sin ^{3}(t)$ for $t \in(0,2 \pi]$. The reason for this assumption lies in the difficulty of guessing the variation of a conductivity function on the boundary of the exterior cylinder in the realistic situations. With this additional perturbation on the scattered field, one can consider the noise level is higher than 3\%.

One of the critical points in the Newton-type reconstruction algorithms is the selection of a criterium when to stop the iterative steps. To do this, we check the difference of the obtained shape update parameters $q$ in successive iterations. The update function $q$ is expressed in terms of $m$ th degree of trigonometric polynomials

$q(t)=\sum_{m=0}^{M}\left(a_{m} \cos m t+b_{m} \sin m t\right)$ for $t \in(0,2 \pi]$ and Eqs. (3.13) and (3.15) are evaluated in the sense of least squares. In this direction, the expression

$\epsilon=\sum_{m=0}^{M} \frac{\left|a_{m}^{(i)}-a_{m}^{(i-1)}\right|}{M+1}+\sum_{m=1}^{M} \frac{\left|b_{m}^{(i)}-b_{m}^{(i-1)}\right|}{M}$

is employed to make a decision on the running iterations. Here, $\epsilon$ is a stopping criterium and $i$ represents the iteration number. We terminate iterations for $\epsilon<10^{-4}$, in IP1 and in IP2.

The choice of the degree of polynomials in Eq. (4.2) can be considered as an additional regularization parameter. We note that regularization parameters $\alpha$ in (3.7), $\beta$ for the equation system (3.14)-(3.17), $\gamma$ for Eq. (3.15) and $m$ in (4.2) are chosen by trial and error as a typical procedure which is applied in [23-28]. However, it is important to emphasize that the selection of the mentioned parameters do not affect the quality of the reconstructions if $\alpha \in\left[10^{-5}-10^{-7}\right], \quad \beta=10^{-5}$ and $M=[4,5]$ for IP1 and $\alpha \in$ $\left[10^{-2}-10^{-3}\right], \gamma=10^{-7}$ and $M=[2,3]$ for IP2 are chosen, see Table 1.

\subsection{Numerical results for IP1}

The motivation for the investigation of this case is based on the analogy between the mathematical statement of IP1 and the identification problem for a small air gap in a tendon duct. Here, the size of the air conclusion is considered comparable with the size of an steel bar inside of the duct. In this scenario, circular cylindrical shaped metal wires $C_{1}, C_{2}, \ldots, C_{6}$ with a radius of $r_{m}=3 \mathrm{~cm}$ have to be taken into account in the problem geometry. We note that locations of these wires are given. To find the location of the air gap, the reconstruction algorithm, which is described in Section 3.1, is applied. We consider a regular square grid $10 \times 10 \mathrm{~cm}$ on the physical domain $D_{1}$, such that the distance between two adjacent grid points is $1.25 \mathrm{~cm}$. Afterwards, center coordinates of the circular cylinder having radius $r_{0}=2 \mathrm{~cm}$ are located at grid points and the remaining steps of the proposed algorithm are employed. We observed $\approx 2.263 \%$ error in the reconstructed coordinates for the first two experiments, although the exact location is obtained for the last test case of IP1. Furthermore, the convergence of the reconstructed locations is checked with a finer mesh whose distance between grid points is $0.125 \mathrm{~cm}$ and in such case $\approx 1.789 \%$ error is observed. In this context, Fig. 2 illustrates the convergence behavior of the method for a fine mesh where the first 10 values of $\min (\xi)$ provide a good accuracy.

From now on we investigate the performance of the shape reconstruction algorithms for the found locations in different cases where the resultant shapes are indicated with red curves in all figures.

In the first experiment we consider, the shape of buried airfilled cavity can be represented by a star-like parametrization with a radius function $r^{a}$, and the conductivity function on $\Gamma_{1}$ is chosen as $\lambda_{1}$. To obtain the reconstruction in Fig. 3, we use $\alpha=10^{-5}$, $\beta=10^{-5}, M=5$ in $i=33$ iterations.

Table 1

Parameters used in the experiments.

\begin{tabular}{llllllll}
\hline Fig. no & $\lambda$ & $\Gamma_{0}$ & \multicolumn{6}{l}{ Simulation parameters } \\
\cline { 3 - 8 } & & & \multicolumn{1}{c}{$i$} & $\alpha$ & $\beta$ & $\gamma$ & $M$ \\
\hline 3 & $\lambda_{1}$ & $r^{a}$ & 33 & $10^{-5}$ & $10^{-5}$ & - & 5 \\
4 & $\lambda_{2}$ & $r^{a}$ & 10 & $10^{-7}$ & $10^{-5}$ & - & 4 \\
5 & $\lambda_{1}$ & $r^{b}$ & 6 & $10^{-5}$ & $10^{-5}$ & - & 5 \\
6a & $\lambda_{1}$ & $r^{c}$ & 12 & $10^{-3}$ & - & $10^{-3}$ & 3 \\
6b & $\lambda_{2}$ & $r^{b}$ & 17 & $10^{-2}$ & - & $10^{-3}$ & 2 \\
\hline
\end{tabular}




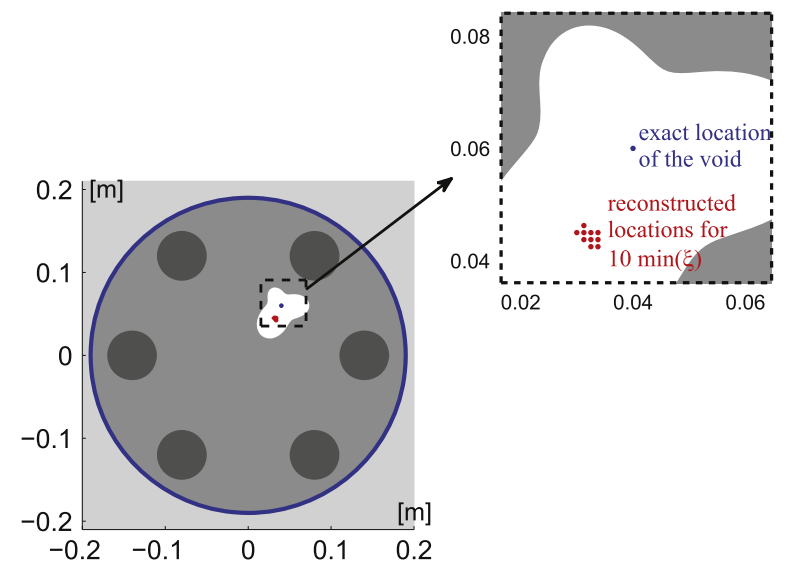

Fig. 2. Reconstructed locations for $\Gamma_{0}=r^{a}(t)(\cos t, \sin t), \lambda=\lambda_{1}$ and a grid resolution $0.125 \mathrm{~cm}$.

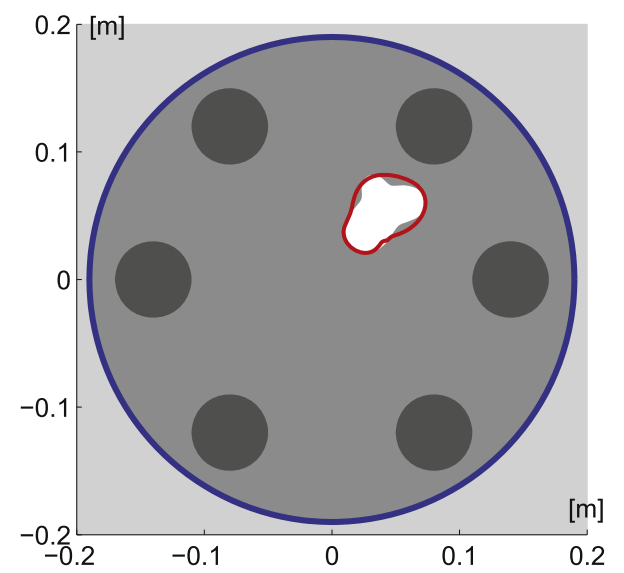

Fig. 3. Identification of an air void in a tendon duct. (For interpretation of the references to color in this figure caption, the reader is referred to the web version of this paper.)

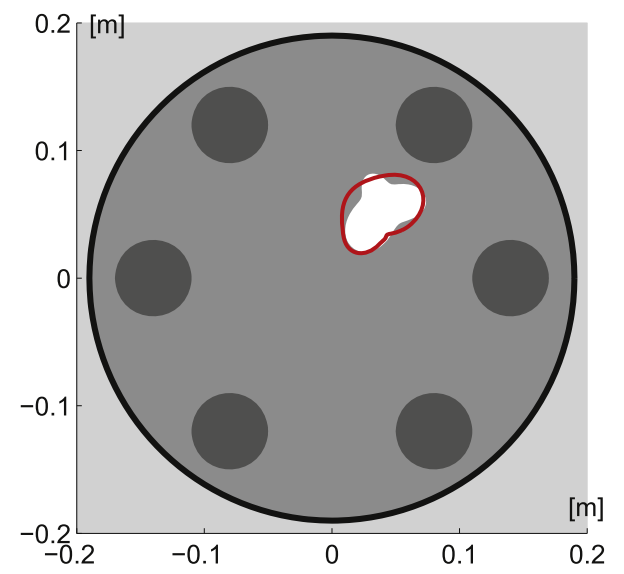

Fig. 4. Identification of an air void in a tendon duct. (For interpretation of the references to color in this figure caption, the reader is referred to the web version of this paper.)

As a second test, we check the quality of the reconstructions by changing the function from $\lambda_{1}$ to $\lambda_{2}$ on $\Gamma_{1}$ and keeping the rest of the simulation parameters the same as initial experiment. In this case, $\alpha=10^{-7}, \beta=10^{-5}, M=4$ are chosen for the regularizations and the shape, presented with red curve in Fig. 4, is found for $i=10$ iterations.

Afterwards, $\Gamma_{0}$ is considered with a radius function $r^{b}$ for $b_{0}=0.05$ and buried at a different location which is more closer

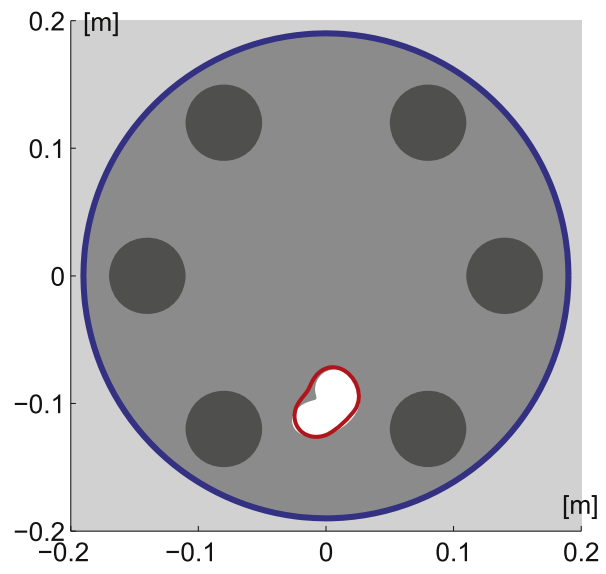

Fig. 5. Identification of an air void in a tendon duct. (For interpretation of the references to color in this figure caption, the reader is referred to the web version of this paper.)

to the metallic wires as compared to the previous two experiments. The function $\lambda=\lambda_{1}$ is used and the same regularization parameters are chosen as in the first experiment. The reconstruction given in Fig. 5 is obtained for $i=6$ iterations.

The numerical results of this section show that the proposed inversion algorithms for the solution of IP1 provide sufficiently stable results for different types of air voids located at different locations under noisy data. Furthermore, it should be added that the norm and the variation of the function $\lambda$ is an important argument on the quality of the reconstructions.

\subsection{Numerical results for IP2}

In this section, it is assumed that tendons are gathered inside of the duct due to overload, aging, etc., which is illustrated in Fig. 2 of Schickert's study [7]. Therefore, the shape of multi-wire metal structure is approximated by a single arbitrarily shaped metallic bar. Our aim is to find the location and shape of this buried metallic structure. Since such type of testing gives an idea on the irregular decomposition of the metallic wires, it can be employed for the inspection of the ducts. This practical application can be modeled by the IP2 according to our formulation. Here, the geometry of problem consists of an object $\Gamma_{0}$, so that transmission conditions are satisfied on its boundary, and it is buried in a cylinder $\Gamma_{1}$ having conductive boundary conditions.

In the solution of IP2, radius of the initially guessed circular cylinder is chosen to be large enough, $r_{0}=17 \mathrm{~cm}$ in order to eliminate the usage of any kind of location reconstruction algorithm.

For the first example, the radius function for $\Gamma_{0}$ is assumed to be $r^{c}$ with $\lambda=\lambda_{1}$ and $\alpha=10^{-3}, \gamma=10^{-3}, M=3$ are chosen. The inversion algorithm run for $i=12$ iterations and provides the result in Fig. 6a. In the last experiment we change the buried metallic structure shape and represent with $r^{b}$ for $b_{0}=1$ where $\lambda=\lambda_{2}$. The regularization parameters $\alpha=10^{-2}, \gamma=10^{-3}, M=2$ are chosen. For $i=17$ the reconstructed shape is illustrated in Fig. $6 \mathrm{~b}$.

In order to summarize all parameters in a compact form and to observe the parameter space in a more clear way we introduce Table 1.

\section{Conclusion}

In this paper, two different inverse problems whose aims are identifications of buried objects in bounded regions are investigated in order to model selected non-destructive testing scenarios 
a

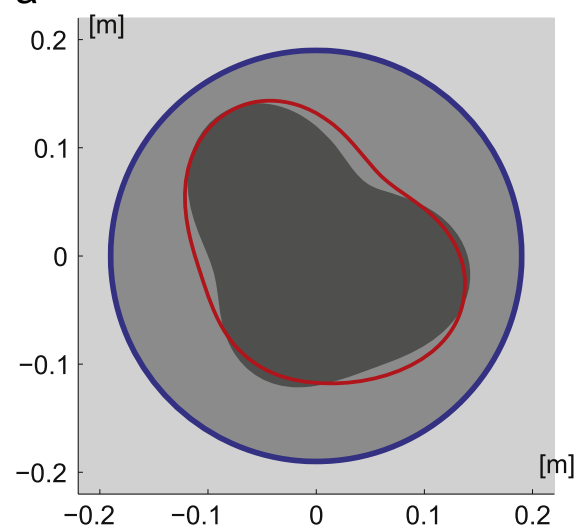

$\mathrm{b}$

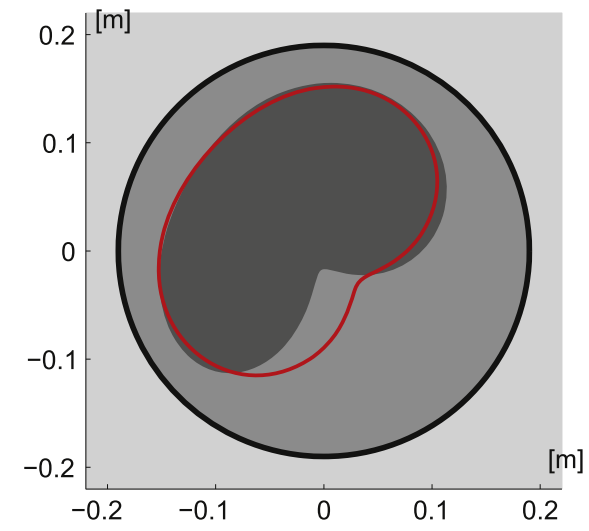

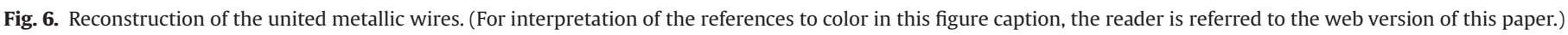

of concrete structures. The proposed numerical reconstruction algorithms are performed on a standard type of personal computer and satisfactory results are obtained in a few minutes. The most time consuming part of the computations is the application of location reconstruction algorithm on finer grids. However, this procedure consists of solution of uncoupled direct scattering problems for each grid points, which can be carried out in parallel computations.

In the current study, we show results of numerical experiments for new problem geometries such that the reconstructions which were presented in $[2,27,28]$ support the verification of the proposed algorithms. It is observed that the localization of the unknown buried objects are succeeded with an acceptable accuracy for different types of objects and conductivity functions.

The selected parameters for the regularizations express the scope of arbitrarily chosen variables can change in a small scale and in some cases they can be chosen the same for different types of buried objects. Furthermore, Tikhonov parameters for the IP2 are chosen stronger and the degrees of polynomials are smaller as compared to IP1. The number of illuminations plays a critical role on the quality of the reconstructions and the stability of the results especially in IP2, therefore we need to use four different angles of incidence. On the other hand, it is still possible to find reasonable reconstructions with two illuminations for the IP1.

The boundary integral equation formulations of the inverse problems are valid also for arbitrarily shaped objects although we consider in the numerical experiments the exterior cylinder and interior metal wires are circular cylinders. However, as it is mentioned in the mathematical statement of the problems that the boundary of the buried object should not be connected with the other boundaries.

As it is noted, we assume that initial processing steps are done and scattered acoustic field on a measurement circle is prepared in this study. For the verifications of simulations with experiments additional effects have to be considered, e.g. polarization, mode conversions, etc.

As a future work we plan to extend our algorithms for buried multi-scatterers. Moreover, reconstruction algorithms can be improved for the cases when there is a lack of information on the size of the buried object. Additionally, we consider the usage of multi-frequency insonifications and the verifications of simulations with real data experiments.

\section{Acknowledgments}

The authors would like to thank to Prof. Dr. K. J. Langenberg for the idea on the application of Newton-type methods to NDT problems. The support of DFG under Grant YA304/2-1 for this study is gratefully acknowledged.

\section{References}

[1] Bore T, Placko D, Taillade F, Sabouroux P. Electromagnetic characterization of grouting materials of bridge post tensioned ducts for NDT using capacitive probe. NDT\&E Int 2013;60:110-20.

[2] Yaman F, Weiland Th. Location and shape reconstructions of objects buried in inhomogeneously penetrable cylinders. In: International conference proceedings on the electromagnetics in advanced applications (ICEAA); 2013. p. 1505-8.

[3] Hellier CJ. Handbook of nondestructive evaluation. second ed.. New York: McGraw-Hill; 2013.

[4] Langenberg KJ, Marklein R, Mayer K. Ultrasonic nondestructive testing of materials, theoretical foundations. Boca Raton: CRC Press, Taylor \& Francis Group; 2012.

[5] Maierhofer C, Reinhardt HW, Dobmann G. Non-destructive evaluation of reinforced concrete structures, non-destructive testing methods, vol. 2. Cambridge: CRC Press, Woodhead Publishing Limited; 2010.

[6] Algernon D, Gräfe B, Mielentz F, Köhler B, Schubert F. Imaging of the elastic wave propagation in concrete using scanning techniques: application for impact echo and ultrasonic echo methods. J Nondestruct Eval 2008;27:83-97.

[7] Schickert M. Ultrasonic NDE of concrete. In: IEEE ultrasonics symposium 2002; 2002. p. 739-48.

[8] Krause M, Millmann B, Schickert M, Mayer K. Investigation of tendon ducts by means of ultrasonic echo methods: a comparative study. In: ECNDT 2006 proceedings-Tu.3.2.1, Berlin; 2006.

[9] Krause M, Gräfe B, Mielentz F, Millmann B, Friese M, Wiggenhauser H, et al. Ultrasonic imaging of post-tensioned concrete elements: new techniques for reliable localization of grouting defects. Concrete repair, rehabilitation and retrofitting II; 2009. p. 521-7.

[10] Schubert F. Basic principles of acoustic emission tomography. In: 26th European conference on acoustic emission testing 2004, DGZfP-proceedings BB 90-CD. Lecture, vol. 58; 2004. p. 575-85.

[11] Schubert F, Marklein R. Numerical computation of ultrasonic wave propagation in concrete using the elastodynamic finite integration technique (EFIT). In: IEEE ultrasonics symposium 2002; 2002. p. 799-804.

[12] Fellinger P, Marklein R, Langenberg KJ, Klaholz S. Numerical modelling of elastic wave propagation and scattering with EFIT-elastodynamic finite integration technique. Wave Motion 1995;21:47-66.

[13] Marklein R, Langenberg KJ, Bärmann R, Brandfass M. Ultrasonic and electromagnetic wave propagation and inverse scattering applied to concrete. In: Thompson DO, Chimenti DE, editors. Review of progress in quantitative nondestructive evaluation, vol. 15. New York: Plenum Press; 1996. p. 1839-46.

[14] Marklein, R. Numerische Verfahren zur Modellierung von akustischen, elektromagnetischen, elastischen und piezoelektrischen Wellenausbreitungsproblemen im Zeitbereich basierend auf der Finiten Integrationstechnik [PhD thesis]; 1997.

[15] Weiland Th. A discretization method for the solution of Maxwell's equations for six component fields. Electron Commun (AEÜ) 1977;31(3):116-20.

[16] Langenberg KJ, Bärmann R, Marklein R, Irmer S, Müller H, Brandfass M, et al. Electromagnetic and elastic wave scattering and inverse scattering applied to concrete. NDT\&E Int 1997;30(4):205-10.

[17] Seydel A. Ultrasonic synthetic aperture focusing techniques in NDT. In: Sharpe RS, editor. Research techniques in nondestructive testing, vol. 6. New York: Academic; 1983.

[18] Doctor SR, Hall TE, Reid LD. SAFT-the evolution of a signal processing technology for ultrasonic testing. NDT Int 1986;19:163-7. 
[19] Schickert M, Krause M, Müller W. Ultrasonic imaging of concrete elements using reconstruction by synthetic aperture focusing technique. J Mater Civil Eng 2003;15(3).

[20] Langenberg KJ, Mayer K, Marklein R. Nondestructive testing of concrete with electromagnetic and elastic waves: modelling and imaging. Cem Concr Compos 2006;28:370-83.

[21] Bozza G, Brignone M, Pastorino M, Piana M, Randazzo A. Integration of linear sampling and Newton-like schemes in inverse scattering. In: APSURSI ' 09. IEEE; 2009.

[22] Agarwal K, Chen X, Pan L, Yeo SP. Multiple signal classification algorithm for non-destructive imaging of reinforcement bars and empty ducts in circular concrete columns. In: General assembly and scientific symposium, 2011 XXXth URSI; 2011. p. 1-4.

[23] Kress R, Rundell W. Nonlinear integral equations and the iterative solution for an inverse boundary value problem. Inverse Probl 2005;21:1207-23.

[24] Kress R. Newton's Method for inverse obstacle scattering meets the method of least squares. Inverse Probl 2003;19:91-104.

[25] Ivanyshyn O, Kress R, Serranho P. Huygens' principle and iterative methods in inverse obstacle scattering. Adv Comput Math 2010;33(4):413-29.
[26] Altundag A, Kress R. An iterative method for a two-dimensional inverse scattering problem for a dielectric. J Inverse Ill-Posed Probl 2012:20:575-90.

[27] Yaman F. Location and shape reconstructions of sound-soft obstacles buried in penetrable cylinders. Inverse Probl 2009;25(6):1-17.

[28] Yaman F. A combination of shape and conductivity reconstructions methods for an inverse boundary value problem. Wave Motion 2010:47(4):253-63.

[29] Gerlach T, Kress R. Uniqueness in inverse obstacle scattering with conductive boundary condition. Inverse Probl 1996;12:619-25.

[30] Yaman F. Numerical solution of an inverse conductive boundary value problem. Radio Sci 2008;43(6):RS6004.

[31] Potthast R. Fréchet differentiability of boundary integral operators in inverse acoustic scattering. Inverse Probl 1994;10:431-47.

[32] Colton D, Kress R. Inverse acoustic and electromagnetic scattering theory. 3rd ed.. New York: Springer; 2013.

[33] Colton D, Kress R. Integral equation methods in scattering theory, Philadelphia: SIAM; 2013, p. 72

[34] Nédélec JC. Acoustic and electromagnetic equations integral representations for harmonic problems. New York: Springer-Verlag, Inc.; 2001.

[35] Kirsch A, Grinberg N. The factorization method for inverse problems. New York: Oxford University Press; 2008. 